\title{
Herzlich willkommen in Wiesbaden
}

\begin{abstract}
$\mathrm{W}$ ir freuen uns sehr, dass Sie unserer Einladung zum diesjährigen ÄDA-Kongress nach Wiesbaden gefolgt sind. Sie finden in dieser Sonderausgabe von Allergo Journal Kurzbeiträge zu den geladenen Vorträgen des Kongresses (ab S. S10) sowie die Abstracts der eingereichten wissenschaftlichen Beiträge (ab S. S70).

Der guten Tradition folgend wird die interdisziplinäre Fortbildung in allen allergologischen Bereichen im Mittelpunkt

„Die Allergologenfamilie lässt sich auch unter den schwierigen politischen Rahmenbedingungen des Jahres 2006 nicht auseinander dividieren." der Wiesbadener Tagung stehen. Aktuelle Entwicklungen in der Dermatologie, $\mathrm{HNO}$ Heilkunde, Pneumologie/Innerer Medizin und Pädiatrie werden von namhaften Experten vorgestellt. Die Themen umfassen das gesamte weite Feld der praktischen Allergologie! Immer steht jedoch der Praxisbezug im Vordergrund.

In diesem Jahr feiern wir mit dem ÄDA-Kongress auch 100 Jahre Allergie: Im Jahre 1906 wurde das Wort „Allergie“ erstmals vom Wiener Kinderarzt Clemens von Pirquet verwendet.

Auch in diesem Jahr haben sich Allergologen und Fachärzte aus Deutschland und dem gesamten deutschsprachigen Raum angemeldet. Persönlich freut uns besonders die erneut interdisziplinär ausgewogene Zusammensetzung der Teilnehmer - ein eindeutiges Zeichen dafür, dass die Allergologenfamilie sich auch unter den schwierigen politischen Rahmenbedingungen im Jahre 2006 nicht auseinander dividieren lässt.
\end{abstract}



Prof. Dr. Ludger Klimek, Zentrum für Rhinologie und Allergologie, Wiesbaden, Vorstandsmitglied des ÄDA

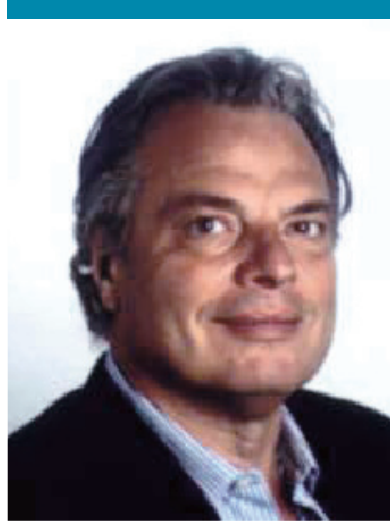

Prof. Dr. Wolfgang W. Schlenter, HNO-Abteilung, St. Marienkrankenhaus, Frankfurt/Main, Schatzmeister des ÄDA
Eine Besonderheit dieser Tagung ist sicherlich die herrliche Umgebung der Veranstaltung in den aufwendig restaurierten Jugendstil-Räumlichkeiten des Kurhauses in Wiesbaden. Der Gesellschaftsabend und eine „After-Work-Party" runden die Tagung ab.

Wir freuen uns, Sie zum AllergieKongress 2006 in Wiesbaden begrüßen zu dürfen!

Mit kollegialen Grüßen $\alpha$



Prof. Dr. Ludger Klimek
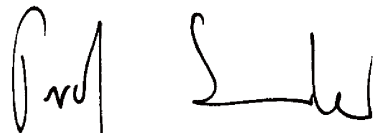

Prof. Dr. Wolfgang W. Schlenter 